\title{
Physiological Activity Depresses Synaptic Function through an Effect on Vesicle Priming
}

\author{
Krista L. Moulder, ${ }^{1}$ Xiaoping Jiang, ${ }^{1}$ Amanda A. Taylor, ${ }^{1}$ John W. Olney, ${ }^{1}$ and Steven Mennerick ${ }^{1,2}$ \\ Departments of ${ }^{1}$ Psychiatry and ${ }^{2}$ Anatomy and Neurobiology, Washington University School of Medicine, St. Louis, Missouri 63110
}

\begin{abstract}
Neurons engage compensatory, homeostatic synaptic changes to maintain their overall firing rate. We examined the induction and expression of a persistent presynaptic adaptation. We explored the effect of mild extracellular potassium elevation to increase hippocampal pyramidal neuron spiking over a physiological range. With several days of mild depolarization, glutamate release adapted, as revealed by an increased mismatch between the number of active, FM1-43-positive, glutamatergic synapses and the total number of synapses defined by vesicular glutamate transporter-1 antibody staining. Surprisingly, the adaptation of glutamate terminals was all-or-none; recycling vesicle pool size at remaining active synapses was not significantly altered by the adaptation. Tetrodotoxin (TTX), but not postsynaptic receptor blockade, reversed depolarization-induced adaptation, and TTX added to normal incubation medium increased the number of active synapses, suggesting that normal spiking activity sustains a steady-state percentage of inactive terminals. Chronic mild depolarization depressed EPSCs and decreased the size of the readily releasable pool of vesicles (RRP). Several hours of $10 \mathrm{~Hz}$ electrical stimulation also depressed the RRP size, confirming that spiking alone induces adaptation and that strong stimulation induces more rapid presynaptic adaptation. Despite the importance of $R R P$ alteration to the adaptation, ultrastructural experiments revealed no changes in docked or total synaptic vesicle numbers. Furthermore, $\alpha$-latrotoxin induced vesicle release at adapted synapses, consistent with the idea that adaptation resulted from changes in vesicle priming. These results show that glutamatergic neurotransmission persistently adapts to changes in electrical activity over a wide physiological range.
\end{abstract}

Key words: homeostasis; synapse; EPSC; glutamate; presynaptic; action potential

\section{Introduction}

Malleability is a long-studied, intriguing property of synapses. Long-term synaptic plasticity, plasticity greatly outliving the inducing stimulus, can be divided into two broad forms: Hebbian and homeostatic (Burrone and Murthy, 2003). Hebbian depression and potentiation of synaptic function are characterized by synapse specificity and associativity. In contrast, homeostatic synaptic plasticity is characterized by global synaptic change in response to changes in the overall activity level of a cell. Induction of homeostatic synaptic change does not necessarily involve correlated or anti-correlated activity in presynaptic and postsynaptic partners. Because it is of opposite polarity to the inducing stimulus, homeostasis may be an important adaptive response to keep positive feedback mechanisms, such as long-term potentiation, from saturating synaptic machinery (Turrigiano and Nelson, 2004). Synaptic homeostasis may also help prevent excessive excitation and excitotoxicity when the balance between excitation and inhibition is upset (Moulder et al., 2004). Of course, these

Received Dec. 22, 2005; revised May 9, 2006; accepted May 10, 2006.

This work was supported by National Institutes of Health Grants NS40488, AA12952, MH78823 (S.M.), DA018109 (K.L.M.), and HD37100 (J.W.O.) and by the MCDonnell Center for Cellular and Molecular Neurobiology (S.M.). We thank Dr. Tamara Hershey, Dr. Malcolm Tobias, and Julian Meeks for help with statistical analyses, Ann Benz for preparation of hippocampal cultures, and all laboratory members for advice and discussion.

Correspondence should be addressed to Dr. Steven Mennerick, Department of Psychiatry, Washington University School of Medicine, 660 South Euclid Avenue, Box 8134, St. Louis, M0 63110. E-mail: menneris@psychiatry.wustl.edu.

D0I:10.1523/JNEUROSCI.5498-05.2006

Copyright $\odot 2006$ Society for Neuroscience $\quad 0270-6474 / 06 / 266618-09 \$ 15.00 / 0$ may be artificial distinctions, and other, hybrid forms of synaptic plasticity may exist. If so, the relevant inducing stimuli and mechanisms of expression of such forms are unclear.

We reported recently that glutamate synapses undergo a persistent presynaptic depression when challenged with strong, brief pathophysiological depolarization (Moulder et al., 2003, 2004). Thus, the response appears homeostatic, or adaptive, but whether physiological activity induces this form of presynaptic plasticity is unknown. The presynaptic change to strong depolarization is reflected in depressed EPSCs evoked in response to hypertonic sucrose, a $\mathrm{Ca}^{2+}$-independent secretagogue thought to trigger selective exocytosis of the readily releasable pool of vesicles (RRP) (Rosenmund and Stevens, 1996). Therefore, we term the synaptic change "persistent RRP depression." In the present work, we show that physiological activity activates this synaptic adaptation bi-directionally, and we investigate the expression mechanisms of this presynaptic depression. We conclude that persistent RRP depression arises from docked vesicles that fail to undergo "priming," the biochemical transition to vesicle release readiness (Sudhof, 2004). Certain synaptic mutants are known to yield priming deficiencies (Augustin et al., 1999), but long-term synaptic modulation by this mechanism has not been described previously. Interestingly, this form of synaptic plasticity primarily affects presynaptic terminals in a binary manner, with many terminals functioning normally and a subset cutoff completely from the ability to exocytose vesicles to physiological (and even nonphysiological) stimuli. Thus, the plasticity 
shares features of both homeostatic plasticity (adaptive, nonassociative) and Hebbian plasticity (synapse specific rather than global).

\section{Materials and Methods}

Materials. Unless otherwise indicated, reagents were purchased from Sigma (St. Louis, MO). $\alpha$-Latrotoxin ( $\alpha$-LTX) was obtained from Alomone Labs (Jerusalem, Israel).

Cell culture. Hippocampal cultures were prepared as described previously (Mennerick et al., 1995). In brief, dissected postnatal (postnatal day $0-3$ ) rat hippocampi were incubated with papain and then mechanically dissociated and plated either at 2000 cells $/ \mathrm{mm}^{2}$ as "mass" cultures or at 100 cells $/ \mathrm{mm}^{2}$ on microdots of collagen as "microisland" cultures. Plating medium consisted of Eagle's medium (Invitrogen, Gaithersburg, MD) supplemented with heat-inactivated horse serum (5\%), fetal bovine serum (5\%), $17 \mathrm{~mm}$ glucose, $400 \mu \mathrm{m}$ glutamine, $50 \mathrm{U} / \mathrm{ml}$ penicillin, and $50 \mu \mathrm{g} / \mathrm{ml}$ streptomycin. Cultures were maintained at $37^{\circ} \mathrm{C}$ in a humidified incubator with $5 \% \mathrm{CO}_{2} / 95 \%$ air. Cytosine arabinoside $(6.7 \mu \mathrm{M})$ was added $3-4 \mathrm{~d}$ after plating to inhibit cell division. At $4-5 \mathrm{~d}$ after plating, a one-half volume medium replacement was conducted using Neurobasal medium (Invitrogen) plus B27 supplement. Subsequent to this medium replacement, neurons were treated with an additional $6 \mathrm{~mm} \mathrm{KCl}$, or 500 nM TTX plus $25 \mu \mathrm{M}$ D-2-amino-5-phosphonovalerate (D-APV) plus $1 \mu \mathrm{M}$ 2,3-dihydroxy-6-nitro-7-sulfonyl-benzo[f]quinoxaline (NBQX). Control neurons received no additions. Experiments were conducted 11-14 d after plating (6-10 $\mathrm{d}$ of treatment).

Electrophysiology. Whole-cell recordings were performed using an Axopatch 1D amplifier (Molecular Devices, Foster City, CA) and a Digidata 1200 acquisition board (Molecular Devices). Electrodes had resistances of 3-5 M $\Omega$, and access resistance was compensated $80-100 \%$. In all instances, cells were excluded from analysis if a leak current $>300 \mathrm{pA}$ was observed.

For recording, the culture medium was exchanged for a saline solution containing (in mM): $138 \mathrm{NaCl}, 4 \mathrm{KCl}, 2 \mathrm{CaCl}_{2}, 1 \mathrm{MgCl}_{2}, 10$ glucose, 10 HEPES, and 0.025 D-APV, pH 7.25. The whole-cell pipette solution contained (in mM): $140 \mathrm{~K}$-gluconate, $0.5 \mathrm{CaCl}_{2}, 5$ EGTA, and $10 \mathrm{HEPES}, \mathrm{pH}$ 7.25. For synaptic recordings, cells were stimulated with $1.5 \mathrm{~ms}$ pulses to $0 \mathrm{mV}$ from $-70 \mathrm{mV}$ to evoke transmitter release (Mennerick et al., 1995).

Solutions were exchanged via a local multibarrel perfusion pipette with a common perfusion port placed within $0.5 \mathrm{~mm}$ of the cell under study. Liquid junction potential measurements exhibit exchange times between barrels of $\sim 50 \mathrm{~ms}$. For hypertonic solution ( $0.5 \mathrm{M}$ sucrose) or 50 $\mu \mathrm{M}$ kainic acid applications, drug was applied for $3 \mathrm{~s}$. Sucrose responses were integrated to include responses beneath the transient peak of the response to $10 \%$ of the steady-state response. Capacitance was estimated as described previously (Xu et al., 2000; Moulder et al., 2002). Miniature EPSCs (mEPSCs) were analyzed with MiniAnalysis version 5.6 (Synaptosoft, Decatur, GA).

Action potentials were measured in the current-clamp recording mode of the patch amplifier. Bicuculline $(50 \mu \mathrm{M})$ was included in the extracellular solution. For threshold measurements, action potentials were generated by injecting depolarizing current (30-200 pA).

For $\alpha$-LTX experiments, neurons were exposed to $0.3 \mathrm{~nm} \alpha$-LTX for 1 $\mathrm{min}$, followed by a $1 \mathrm{~min}$ wash in recording saline. $\alpha$-LTX-induced mEPSCs were then recorded for the next $2 \mathrm{~min}$ in the presence of $1 \mu \mathrm{M}$ TTX. A naive culture dish was used for each recording to prevent crosscontamination with $\alpha$-LTX.

Control and experimental conditions were always performed on sibling cultures from the same litter and plating and on the same day of recording. Solitary microisland neurons were used for all electrophysiology experiments, with the exception of the sucrose responses recorded after field stimulation. In those experiments, sucrose responses were recorded from neurons in mass cultures, with $50 \mu \mathrm{M}$ bicuculline included in the extracellular solution to eliminate any contribution from GABAergic neurons.

Field stimulation. Field stimulation was performed using an RC21BRFS chamber (Warner Instruments, Hamden, CT) and a model S4K stimulator (Grass-Telefactor, West Warwick, RI). Mass cultures plated on $25 \mathrm{~mm}$ coverslips were stimulated with $1 \mathrm{~ms}$ current pulses, yielding a field of $10 \mathrm{~V} / \mathrm{cm}$, at $10 \mathrm{~Hz}$ for $16 \mathrm{~h}$. Cultures were maintained at $37^{\circ} \mathrm{C}$ in a humidified incubator with $5 \% \mathrm{CO}_{2} / 95 \%$ air during stimulation. Control cultures were placed in a similar chamber in the same incubator as the stimulated cultures. D-APV $(25 \mu \mathrm{M})$ and $1 \mu \mathrm{M}$ NBQX were added to both conditions during the incubation period to prevent excitotoxicity.

FM1-43 imaging and immunocytochemistry. Mass cultures plated on coverslips were used for all imaging experiments. Active synapses were labeled with a $2 \mathrm{~min}$ application of $10 \mu \mathrm{M}$ FM1-43FX (Invitrogen) and 45 $\mathrm{mm} \mathrm{K} \mathrm{K}^{+}$(equimolar substitution for $\mathrm{Na}^{+}$) in extracellular recording saline containing $50 \mu \mathrm{M}$ D-APV and $1 \mu \mathrm{M}$ NBQX. Cultures were washed for $10 \mathrm{~s}$ with extracellular recording saline containing $500 \mu \mathrm{M}$ Advasep-7 (CyDex, Overland Park, KS) (Kay et al., 1999) and then in saline alone for $10 \mathrm{~min}$. Cultures were fixed in $4 \%$ paraformaldehyde $/ 0.2 \%$ glutaraldehyde in PBS for 10 min.

After fixation, cells were washed with PBS and exposed to blocking solution (10\% normal goat serum/0.05\% Triton X-100 in PBS) for 15 min. Cells were incubated with vesicular glutamate transporter-1 (vGluT-1) antibody (Chemicon, Temecula, CA) diluted at 1:2500 in blocking solution for $4 \mathrm{~h}$. After primary antibody incubation, cells were washed with PBS and then incubated with cyanine 3 (Cy3)-conjugated anti-guinea pig antibody (1:200 in blocking solution; Chemicon) for 30 min. Coverslips were then washed with PBS and mounted with Fluoromount-G (Southern Biotechnology, Birmingham, AL).

For vGluT-1/glutamate receptor 2 (GluR2) colabeling, cells were fixed with $100 \%$ cold methanol for $20 \mathrm{~min}$. Blocking solution consisted of $10 \%$ normal goat serum $/ 0.1 \%$ Triton X-100 in PBS. vGluT-1 antibody (1: 2500) and GluR2 antibody (1:100; Chemicon) were applied simultaneously overnight. Secondary antibodies were Alexa 488-conjugated anti-guinea pig antibody (1:100; Invitrogen) and Cy3-conjugated antirabbit antibody (1:100; Chemicon).

For GluR1 labeling, cells were fixed with $4 \%$ paraformaldehyde/ $0.2 \%$ glutaraldehyde in PBS for 5 min. Blocking solution consisted of $10 \%$ normal goat serum $/ 0.1 \%$ Triton $\mathrm{X}-100$ in PBS. GluR2 antibody (1:16,000; Upstate, Charlottesville, VA) was applied overnight. Biotinylated goat anti-rabbit antibody (Vector Laboratories, Burlingame, CA) was applied for $30 \mathrm{~min}$, followed by tyramide signal amplification (PerkinElmer, Boston, MA). Cy3-streptavidin (1:500) was used to visualize the signal.

Confocal imaging was performed using a $60 \times$ objective (numerical aperture, 1.4), a C1 scanning confocal laser attached to an inverted Eclipse TE300 microscope (Nikon, Melville, NY), and Z-C1 software (Nikon). A naive observer acquired images of representative fields in $z$-stack using alternating excitation by the 488 and $543 \mathrm{~nm}$ laser lines. Gain settings, dwell time, field of view size, and $z$-stack parameters were kept constant for all images within an experiment. Monochrome images were converted into projected images and analyzed using MetaMorph software (Universal Imaging Corporation, Downingtown, PA). Ten puncta per field and 5-10 fields per condition were analyzed for each experiment. For isolating and testing the FM1-43 fluorescence intensities of the active synapses in the histograms from Figure 3, $E$ and $F$, we analyzed the values falling within the modal peak of the high-intensity component of the bimodal distribution and included values falling within the two bins adjacent to the modal peak. This strategy minimized contribution to the analysis from inactive synapses and allowed us to test differences in the means of fluorescence intensity of active synapses using an unpaired $t$ test. Similar mean values for each peak were obtained by fitting the histograms with a double Gaussian distribution.

Electron microscopy. EPSCs were recorded from isolated neurons (in microisland cultures) to verify glutamatergic phenotype. These neurons were then marked, and cultures were fixed for $1 \mathrm{~h}$ in $3 \%$ glutaraldehyde/ $\mathrm{PBS}, \mathrm{pH} 7.4$ at $4^{\circ} \mathrm{C}$. Cultures were rinsed twice in PBS and incubated in $1 \% \mathrm{OsO}_{4}, 0.8 \%$ potassium ferricyanide in $0.1 \mathrm{M}$ phosphate buffer for 30 $\mathrm{min}$ at room temperature. After rinsing with distilled water, specimens were stained with $2 \%$ aqueous uranyl acetate for $30 \mathrm{~min}$, dehydrated in ethanol, and embedded in poly/bed 812 for $24 \mathrm{~h}$. Sections were cut at $50-70 \mathrm{~nm}$ and poststained with uranyl acetate and lead citrate. Synapses were photographed at $36,000 \times$ on a JEM-100CXII transmission electron microscope (Jeol, Tokyo, Japan). Two independent raters, both naive to 
the experimental conditions, scored the number of docked vesicles and the total number of vesicles in each synapse. Docked vesicles were defined as being adjacent to the postsynaptic density and within either one vesicle diameter from the plasma membrane, or touching the plasma membrane (see Results).

Data acquisition and statistics. pClamp software, version 9 (Molecular Devices), was used for electrophysiology data acquisition and analysis for all experiments. Data plotting was done with SigmaPlot software (SPSS, Chicago, IL). Data are presented in the figures and the text as mean \pm SEM. Paired and unpaired $t$ tests and $\chi^{2}$ tests were used to evaluate statistical significance, as noted in Results. A two-sample power analysis for proportions was used to evaluate the likelihood of a type II error in measurements of docked vesicles.

\section{Results}

Increases and decreases in physiological activity induce presynaptic inactivation

We have demonstrated previously that strong, tonic depolarization, such as that achieved during pathophysiological events, leads to a persistent presynaptic adaptation at glutamate synapses (Moulder et al., 2003, 2004). This form of adaptation is unusual compared with other forms of long-term presynaptic and postsynaptic changes in that it involves binary loss of function at glutamate terminals to both $\mathrm{Ca}^{2+}$-dependent and $\mathrm{Ca}^{2+}$. independent secretagogues (Moulder et al., 2004). Whether this form of adaptation occurs in response to physiological activity is unknown. To determine this, we moved neuronal activity in both directions from baseline in dissociated cultures of postnatal hippocampal neurons.

First, we hypothesized that if persistent adaptation of the RRP occurs with normal activity, we should observe an increase in the percentage of functional synapses with activity blockade. We challenged cells with combined TTX and ionotropic glutamate receptor blockers for 6-10 d to silence electrical activity $(500 \mathrm{nM}$ TTX plus $25 \mu \mathrm{M}$ D-APV plus $1 \mu \mathrm{M}$ NBQX). We assayed persistent RRP adaptation by testing for the signature mismatch between FM1-43 uptake and vGluT-1 immunoreactivity (Moulder et al., 2004), an assay that avoids ambiguities created by assaying presynaptic function with electrophysiological postsynaptic receptor-based assays. We stimulated uptake of FM1-43FX (a fixable form of FM1-43) with strong depolarization $\left(45 \mathrm{mM} \mathrm{K}^{+}\right.$ for $2 \mathrm{~min}$ ), followed by fixation and labeling with vGluT-1 antibody to identify all glutamatergic presynaptic elements. In confocal images in which we examined the intensity of FM1-43FX labeling of vGluT-1-positive puncta, we found that in untreated cultures, $20-30 \%$ of vGluT-1-positive terminals were devoid of FM1-43FX uptake (Fig. 1A,C). Similar percentages have been observed by others (Rosenmund et al., 2002; Altrock et al., 2003). In activity-blocked cultures, we found an increase in the percentage of functional presynaptic elements (Fig. $1 B, C$ ). This is the opposite polarity of the change we previously observed with brief, strong depolarization. The result strongly suggests that the fraction of inactive presynaptic elements observed in our (Moulder et al., 2004) and others' (Rosenmund et al., 2002; Altrock et al., 2003) previous studies represent a class of presynaptic elements rendered functionally silent by basal neuronal activity.

In addition to the change in the percentage of functional synapses, activity-blocked cultures exhibited a small but significant increase in the area of FM1-43 puncta (Fig. $1 D$ ). This is consistent with previous work suggesting a change in synapse size with activity block (Murthy et al., 2001). The average intensity of FM1-43 puncta was also increased from $1691 \pm 43$ (control) to $1769 \pm 50$ (TTX) $(p<0.002)$.

To test whether presynaptic function can be further depressed
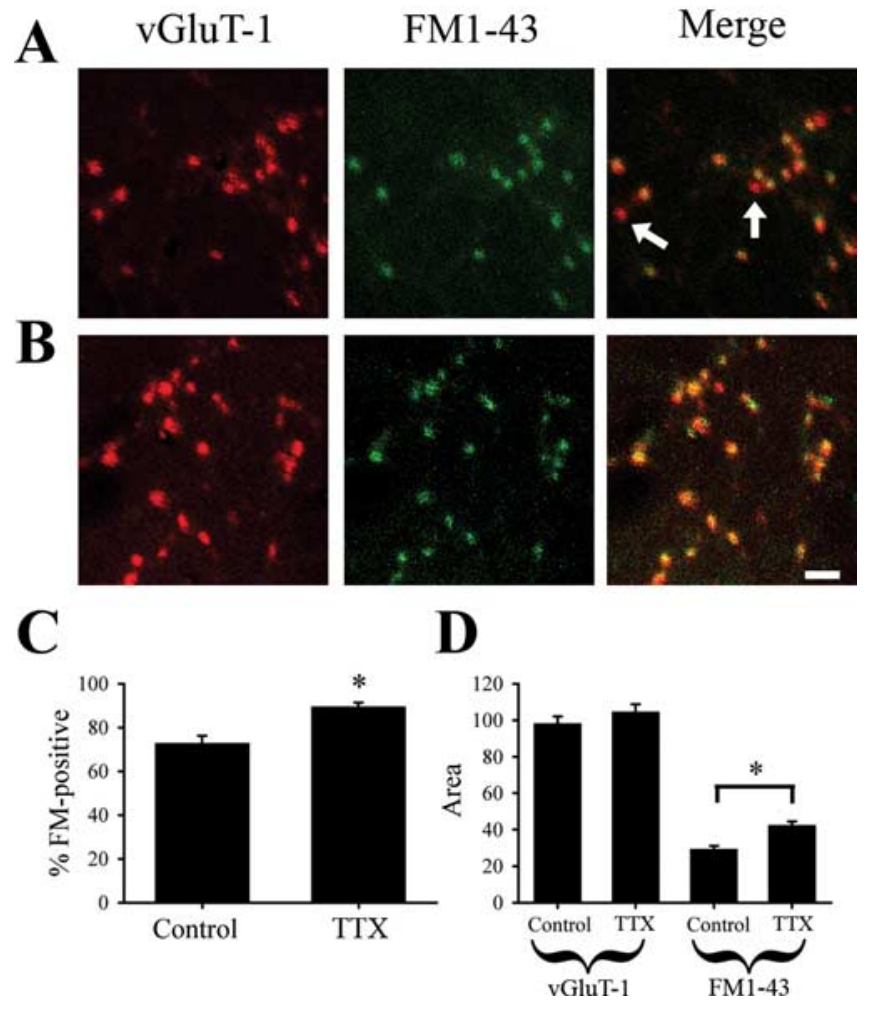

Figure 1. Inactive synapses are decreased by chronic inactivity. $\mathbf{A}$, Control synapses labeled with FM1-43 to identify active synapses and with post hoc vGluT-1 immunofluorescence to identify glutamatergic synapses. The arrows indicate inactive glutamate synapses (VGluT-1 positive, FM1-43 negative). $\boldsymbol{B}$, Similar panels from a culture treated with TTX and glutamate receptor blockers. Scale bar, $5 \mu \mathrm{m}$. C, Summary of the percentage of active synapses from fields obtained in blinded analyses. FM, FM1-43. ${ }^{*} p<0.001$. D, Summary of the quantitative analysis of the synaptic area, as determined by vGluT-1 immunoreactivity or by FM1-43 labeling. A significant increase was observed in the size of FM1-43 puncta after chronic inactivity. ${ }^{*} p<$ 0.001 . For both $\boldsymbol{C}$ and $\boldsymbol{D}, n=6$. Error bars represent SEM.

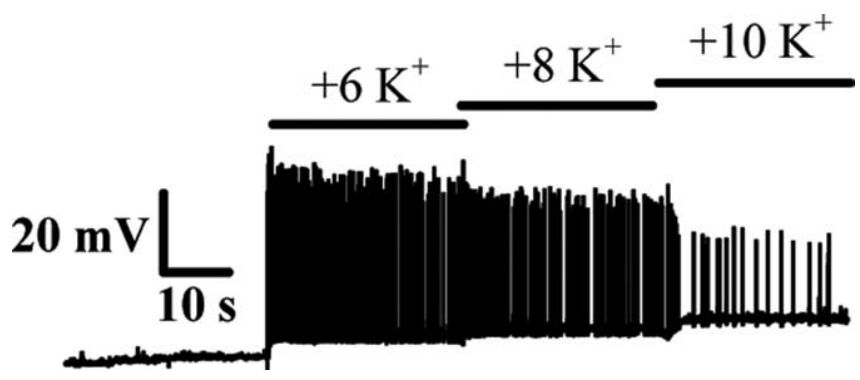

Figure 2. An increase of $6 \mathrm{~mm}\left[\mathrm{~K}^{+}\right]_{0}$ increases spiking acutely and brings neurons closer to threshold chronically. Representative trace of a current-clamp recording during acute application of an additional 6,8 , and $10 \mathrm{~mm}\left[\mathrm{~K}^{+}\right]_{0}$. Note the step depolarization in resting membrane potential and the increase in spiking with $6 \mathrm{~mm}\left[\mathrm{~K}^{+}\right]_{0}$.

from baseline by increased activity, we titrated extracellular $\mathrm{K}^{+}$ concentration $\left(\left[\mathrm{K}^{+}\right]_{\mathrm{o}}\right)$ to yield an increase in spontaneous spike frequency. Acutely, we found that an increase of $6 \mathrm{~mm}\left[\mathrm{~K}^{+}\right]_{\mathrm{o}}$ (from basal $4 \mathrm{~mm}$ ) increased spike frequency from $0.51 \pm 0.26$ to $1.69 \pm 0.53 \mathrm{~Hz}$ (Fig. 2), still well within the range of normal spontaneous spiking activity for pyramidal cells in situ (Gao et al., 1998, 1999). Chronically, by the end of a 6-9 d treatment, we found that resting membrane potential was only $10.7 \pm 0.6 \mathrm{mV}$ away from spike threshold, compared with $16.7 \pm 1.3 \mathrm{mV}$ in control neurons $(n=8$ for each; $p<0.001)$, confirming the increased excitability of treated cells over the period of exposure. 


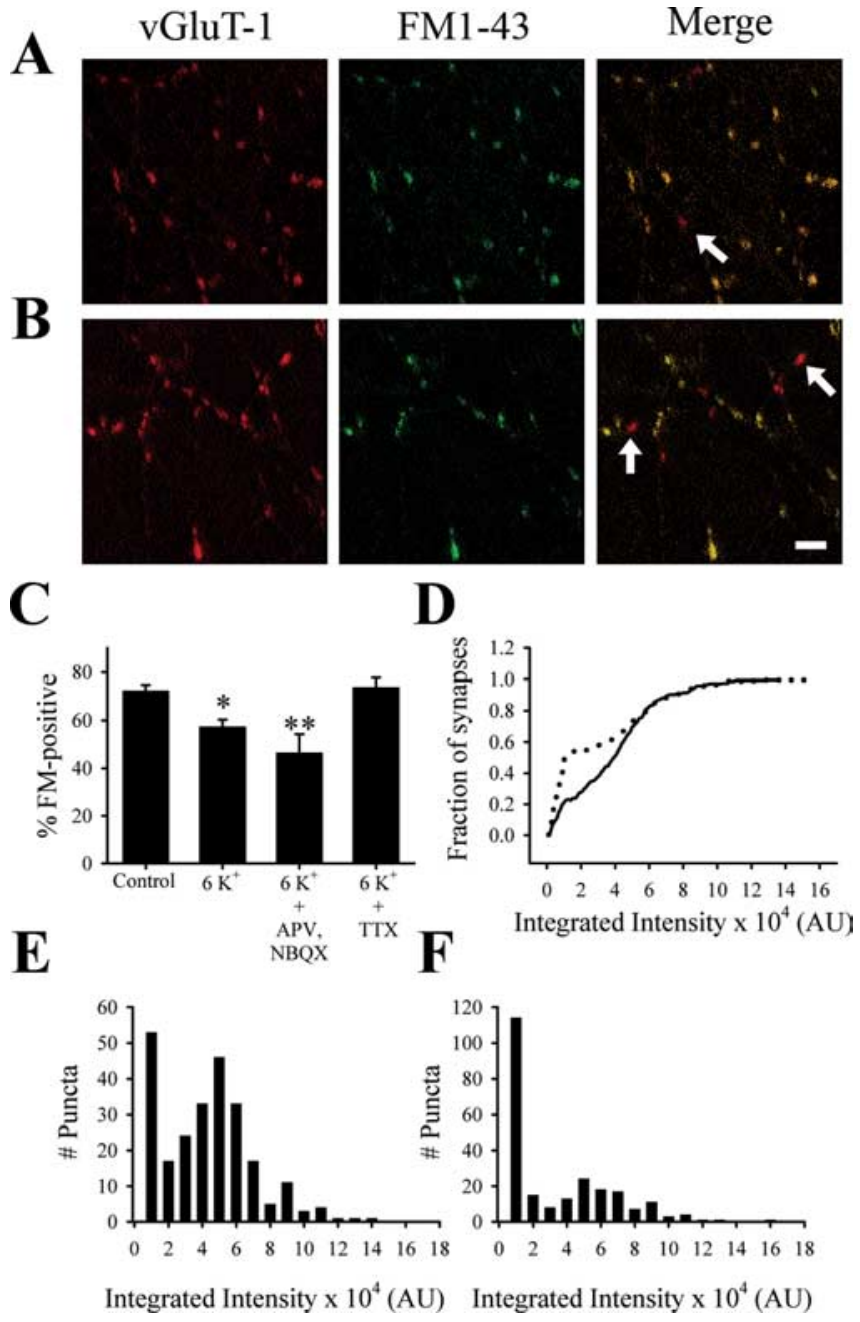

Figure 3. Inactive synapses are increased by chronic exposure to slightly elevated $\left[\mathrm{K}^{+}\right]_{0} . \boldsymbol{A}$, Control synapses labeled with FM1-43 to identify active synapses and with post hoc vGluT-1 immunofluorescence to identify glutamatergic synapses. The arrows indicate inactive glutamate synapses (vGluT-1 positive, FM1-43 negative). $\boldsymbol{B}$, Similar panels from a culture treated with an additional $6 \mathrm{~mm}\left[\mathrm{~K}^{+}\right]_{0}$. Scale bar, $5 \mu \mathrm{m}$. $\boldsymbol{C}$, Summary of the percentage of active synapses from fields obtained in blinded analyses $(n=3-12) .{ }^{*} p<0.001$ and ${ }^{* *} p<0.002$ compared with control. FM, FM1-43. D, Cumulative histograms of FM1-43 puncta integrated intensities for $>200$ terminals (defined by vGluT-1 immunoreactivity) in each group from a single experiment. The solid line represents puncta from control cultures, and the dotted line represents puncta from $\mathrm{K}^{+}$-treated cultures. $\boldsymbol{E}, \boldsymbol{F}$, Histograms of the integrated intensity (area $X$ intensity) values for individual FM1-43 puncta from control $(\boldsymbol{E})$ or $\mathrm{K}^{+}$-treated $(\boldsymbol{F})$ cultures. $\boldsymbol{E}$ and $\boldsymbol{F}$ contain binned data from the same terminals represented by the cumulative plots in $\boldsymbol{D}$. Bin size was 10,000 arbitrary units (AU). Note the two populations of synapses. Histograms are not background subtracted, accounting for the nonzero initial peak representing inactive terminals. Note the change in $y$-axis between $\boldsymbol{E}$ and $\boldsymbol{F}$. Error bars represent SEM.

After brief $16 \mathrm{~h}$ incubation with $6 \mathrm{~mm}\left[\mathrm{~K}^{+}\right]_{\mathrm{o}}$, we induced no detectable synaptic adaptation (data not shown). This is a time frame over which $30 \mathrm{~mm}\left[\mathrm{~K}^{+}\right]_{\mathrm{o}}$ induces strong presynaptic adaptation (Moulder et al., 2004). However, we found that chronic (6-9 d) exposure to the mild depolarization induced highly reliable depression of the percentage of vGluT-1-positive puncta with corresponding FM1-43 fluorescence (Fig. 3A-C). Furthermore, we found that blockade of synaptic glutamate receptors (25 $\mu \mathrm{M}$ D-APV plus $1 \mu \mathrm{M}$ NBQX) failed to alter the decrease in functional presynaptic elements (Fig. 3C). This result is consistent with the idea that postsynaptic receptor activity is not required for this form of synaptic plasticity. On the other hand, $500 \mathrm{~nm}$ TTX prevented the depolarization-induced decrease in the per- centage of functional terminals (Fig. 3C), demonstrating that spiking behavior, rather than the change in resting membrane potential, was important for the effect of increased $\left[\mathrm{K}^{+}\right]_{\mathrm{o}}$. The decrease in functional presynaptic sites was the primary change detected in these FM1-43/vGluT-1 colabeling experiments, because no statistically significant differences were measured in the area or intensity of puncta. For vGluT-1 puncta, average area was $93.4 \pm 10.2$ (control) and $76.4 \pm 3.9\left(\mathrm{~K}^{+}\right)(p<0.380)$, and average intensity was $1841 \pm 574(\mathrm{control})$ and $1636 \pm 424\left(\mathrm{~K}^{+}\right)$ ( $p<0.508$ and $n=6$ experiments). For FM1-43 puncta, average area was $67.2 \pm 14.6(\mathrm{control})$ and $48.7 \pm 9.8\left(\mathrm{~K}^{+}\right)(p<0.143)$, and average intensity was $1588 \pm 397$ (control) and $1420 \pm 390$ $\left(\mathrm{K}^{+}\right)(p<0.063$ and $n=6$ experiments $)$. Overall, these experiments show that decreases and increases in spiking activity over the physiological range alter the percentage of functional presynaptic elements at glutamate synapses, without clearly altering the number of functionally available vesicles remaining at active terminals.

To address the apparent bimodality of the presynaptic change in function further, we examined a large number of terminals in a set of three experiments, so that we could construct histograms of staining intensity. Data from one of these experiments are illustrated in Figure 3. Control terminals exhibited a distribution of staining intensity that was clearly bimodal, demonstrable in both cumulative probability histograms (Fig. $3 D$, solid line) and conventional histograms (Fig. $3 E$ ). The first peak of the distribution did not exceed background levels of fluorescence, obtained from cellular regions adjacent to vGluT-1-positive puncta (data not shown). Therefore, we interpret this peak to represent inactive terminals. The second peak of the integrated intensity distribution was broader, presumably reflecting the range of vesicle numbers constituting the recycling pool at different hippocampal synapses (Harata et al., 2001). When we examined the terminals in depolarized cultures (Fig. 3D, dotted line, F), we found that the main difference from control was a change in the relative amplitude of the two peaks (Fig. $3 E, F$ ), with no change in the means of the two peaks: $6389 \pm 368$ fluorescence units (control, first peak), $6291 \pm 230$ fluorescence units $\left(\mathrm{K}^{+}\right.$, first peak; $\left.p<0.814\right)$, $49,911 \pm 2571$ fluorescence units (control, second peak), and $55,362 \pm 1259$ fluorescence units $\left(\mathrm{K}^{+}\right.$, second peak; $\left.p<0.166\right)$. Similar results were obtained with histograms from two other experiments in which $>200$ FM1-43 puncta were examined per condition (data not shown). These results are consistent with the hypothesis that the persistent synaptic change represents primarily a binary change in function at a subset of glutamate synapses. The results match our conclusions from independent experiments on smaller numbers of terminals and previous experiments using very strong stimulation (Moulder et al., 2004).

We tested whether postsynaptic function was also altered by these prolonged treatments. We found that a $6 \mathrm{~mm}$ increase in $\left[\mathrm{K}^{+}\right]_{\mathrm{o}}$ had no effect on the percentage of GluR2 puncta apposed to vGluT-1 puncta (Fig. $4 A-C$ ), suggesting no increase in the number of postsynaptically silent synapses. The size (Fig. 4D) and average intensity (data not shown) of GluR2 puncta were also unchanged, consistent with the idea that postsynaptic receptor density was not affected. In separate experiments, we also observed no change in the size or average intensity of GluR1 puncta (data not shown).

\section{Electrophysiological tests of adaptation to mild depolarization}

To complement imaging experiments that suggested presynaptic changes with no evidence of accompanying postsynaptic change, 


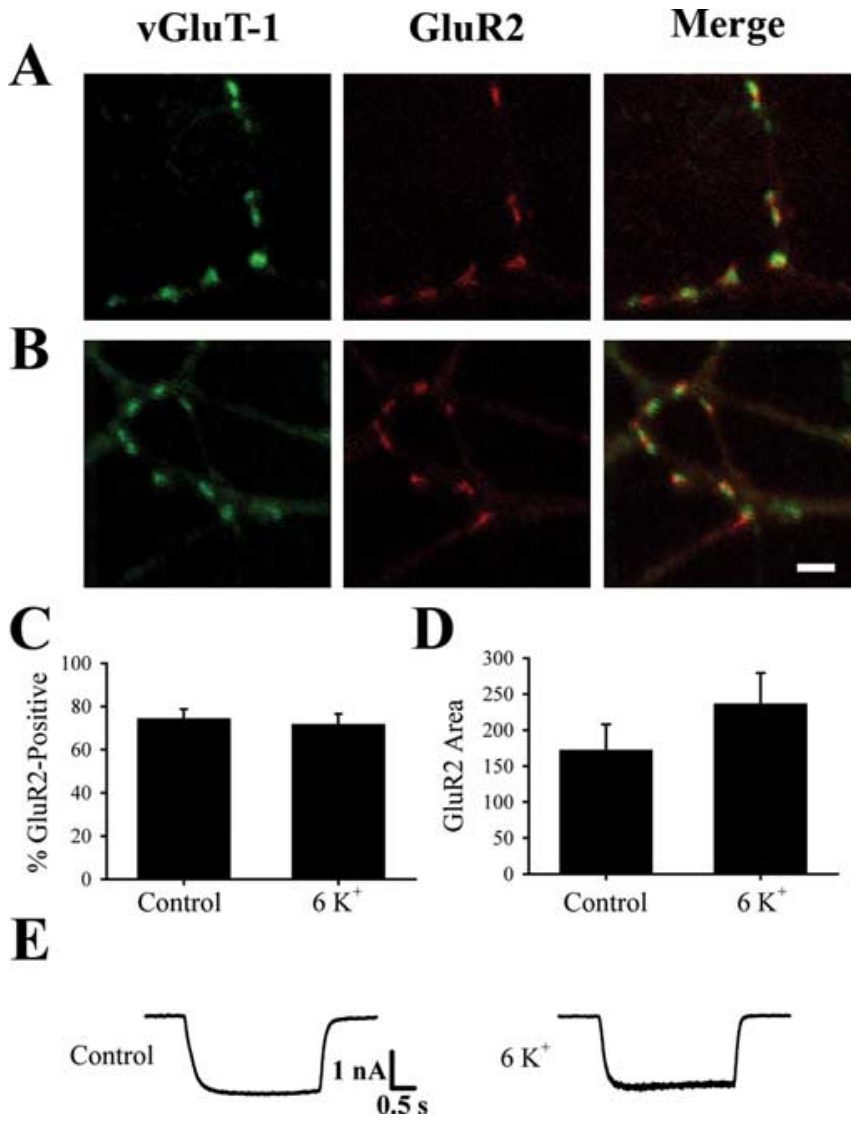

Figure 4. Chronic exposure to slightly elevated $\left[\mathrm{K}^{+}\right]_{0}$ does not alter postsynaptic glutamate receptors. $A$, Control synapses coimmunostained for vGluT- 1 and GluR2. B, Similar panels from a culture treated with an additional $6 \mathrm{~mm}\left[\mathrm{~K}^{+}\right]_{0}$. Scale bar, $5 \mu \mathrm{m}$. C, Summary of the percentage of vGluT-1-positive sites that colabeled with GluR2. D, Summary of the area of GluR2positive puncta. For $\boldsymbol{C}$ and $\boldsymbol{D}$, no statistically significant differences were observed. $n=3$ for each. $\boldsymbol{E}$, Representative traces after exogenous application of $50 \mu \mathrm{m}$ kainic acid from a control neuron and $\mathrm{K} \mathrm{K}^{+}$-treated neuron. The capacitance of the control cell was $22.7 \mathrm{pF}$ and that of the $\mathrm{K}^{+}$-treated cell was $24.3 \mathrm{pF}$. Error bars represent SEM.

we performed electrophysiological tests of the locus of change. After 6-9 d exposure to the mildly depolarizing conditions, we examined electrophysiological responses to exogenous application of the AMPA/KA receptor antagonist kainic acid. We found no change in current density (Fig. $4 E$ ), with an average of $-53.2 \pm 7.0 \mathrm{pA} / \mathrm{pF}$ in control neurons and $-45.4 \pm 3.7 \mathrm{pA} / \mathrm{pF}$ in $\mathrm{K}^{+}$-treated neurons $(n=15 ; p<0.34)$. Therefore, the major changes in function in response to activity changes under our conditions are presynaptic.

Given that immature presynaptic elements can exist with no postsynaptic partner (Ahmari et al., 2000) and that these immature profiles might contribute to the mismatched FM1-43/ vGluT-1 staining, we tested whether the functionally inactivated terminals (Fig. 3) have an impact on signaling by performing electrophysiology. We found that action-potential-evoked autaptic EPSCs were depressed by $6-9 \mathrm{~d}$ of treatment with the $6 \mathrm{~mm}$ $\left[\mathrm{K}^{+}\right]_{\mathrm{o}}$ increase from $-6143 \pm 1266 \mathrm{pA}$ (control) to $-1780 \pm$ $512 \mathrm{pA}(n=9$ for each; $p<0.006)$ (Fig. $5 A, D)$. Both FM1-43 experiments and these physiology experiments were performed with $\mathrm{Ca}^{2+}$-dependent transmitter release. To test whether $\mathrm{Ca}^{2+}$ independent secretagogues also yield depressed release, we used hypertonic sucrose $(0.5 \mathrm{M})$, which causes exocytosis of the RRP (Rosenmund and Stevens, 1996). Sucrose responses were depressed from $-980.9 \pm 202.4 \mathrm{pC}$ (control) to $-436.7 \pm 78.1 \mathrm{pC}$

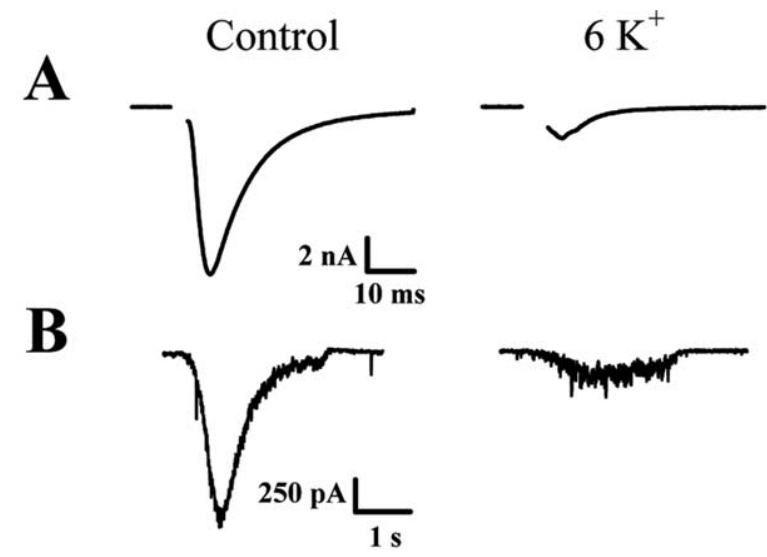

C

Control

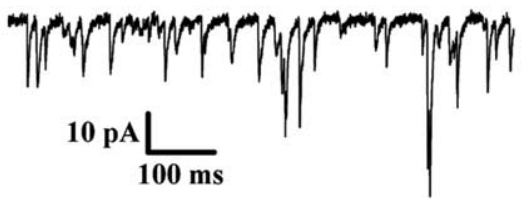

$6 \mathrm{~K}^{+}$

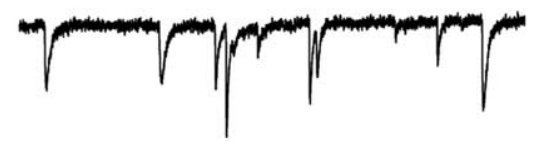

D

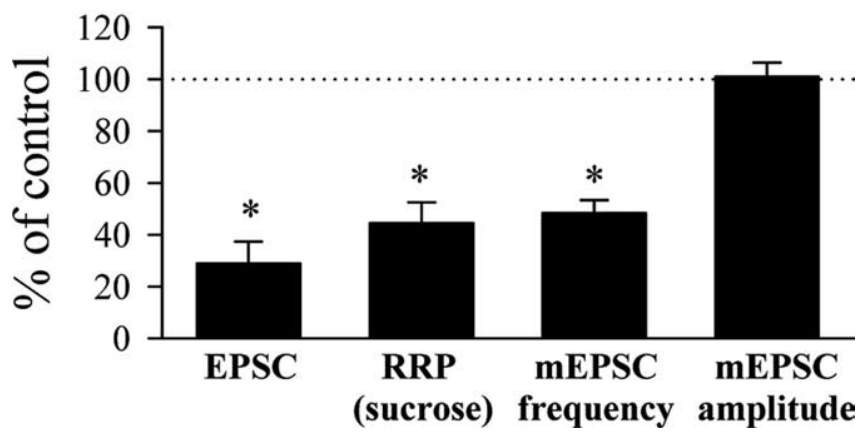

Figure 5. Chronic mild depolarization depresses glutamatergic synaptic transmission. $A$, Representative electrically evoked autaptic EPSCs from solitary neurons maintained in control medium or after chronic treatment with an additional $6 \mathrm{~mm}\left[\mathrm{~K}^{+}\right]_{0}$. B, Representative sucroseevoked currents from the same neurons as in $\boldsymbol{A}$. C, Sample traces showing mEPSCs elicited by 0.3 m sucrose application and recorded in control or $\mathrm{K}^{+}$-treated neurons. D, Summary of the effect of chronic mild depolarization on EPSC amplitude, RRP size, and mEPSC frequency and amplitude. All values are normalized to control values from the same experiment. Raw values for each are presented in Results. ${ }^{*} p<0.02$. Error bars represent SEM.

( $n=9$ for each; $p<0.018)$, similar to action potential responses (Fig. $5 B, D$ ), indicating that stages of presynaptic function adapting to activity are not related to $\mathrm{Ca}^{2+}$ influx. To test whether the depressed RRP size resulted from a change in the rate of vesicle pool recovery, we induced glutamate release with paired sucrose applications delivered $3.5 \mathrm{~s}$ apart to measure relative recovery of the sucrose-accessible RRP (Altrock et al., 2003). RRP size recovered to $43.8 \pm 2.5$ and $40.0 \pm 3.0 \%$ in control and $\mathrm{K}^{+}$-treated neurons, respectively ( $p<0.331$ and $n=11$ for each), indicating that chronic mild depolarization had no effect on the kinetics of RRP refilling.

We also evaluated mEPSCs after chronic mild depolarization. mEPSCs were elicited with $0.3 \mathrm{~m}$ sucrose (Rosenmund and Stevens, 1996; Moulder et al., 2004). In 15 neurons examined for each treatment group, we found that the frequency $(23.3 \pm 1.5$ 

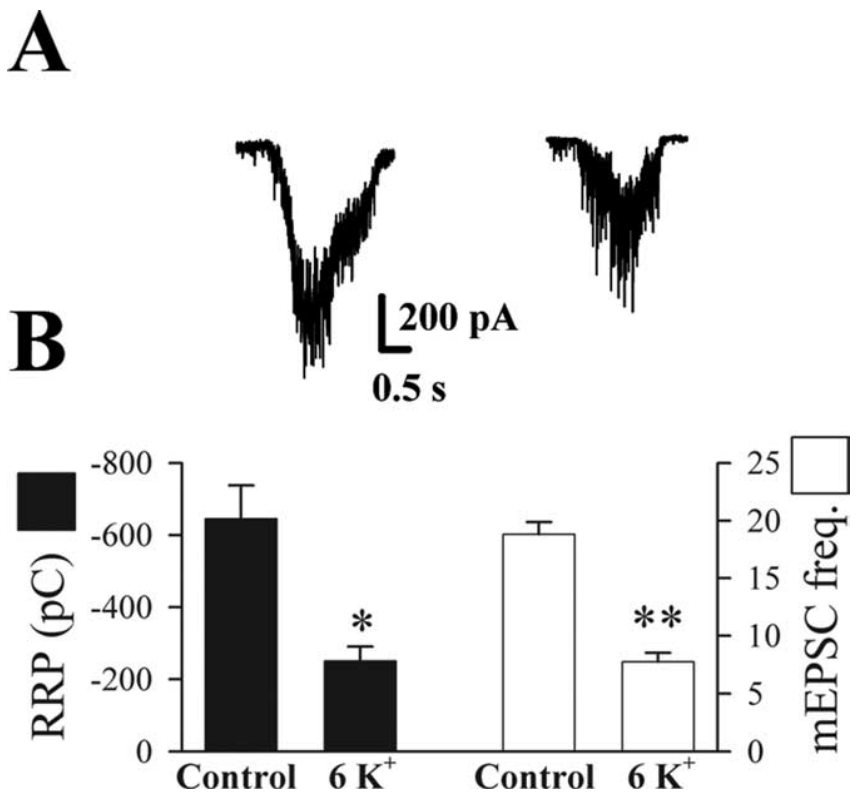

Figure 6. Sustained electrical stimulation depresses glutamatergic signaling. $\boldsymbol{A}$, Representative sucrose-evoked currents from an unstimulated neuron (left) and from a neuron exposed to chronic $10 \mathrm{~Hz}$ field stimulation (right). $\boldsymbol{B}$, Summary of the effect of chronic stimulation on the total charge of sucrose-evoked currents $\left(n=13 ;{ }^{*} p<0.02\right)$ and the frequency (freq.) of $\mathrm{mEPSC}$ elicited by $0.3 \mathrm{~m}$ sucrose application $\left(n=9 ;{ }^{* *} p<0.001\right)$. Error bars represent SEM.

$\mathrm{Hz}$ for control; $11.3 \pm 1.2 \mathrm{~Hz}$ for $\left.\mathrm{K}^{+} ; p<0.001\right)$, but not the amplitude (20.7 $\pm 1.2 \mathrm{pA}$ for control; $21.0 \pm 1.1 \mathrm{~Hz}$ for $\mathrm{K}^{+} ; p<$ 0.88 ), of mEPSCs was decreased by $6-9 \mathrm{~d}$ of treatment with the 6 $\mathrm{mm}\left[\mathrm{K}^{+}\right]_{\mathrm{o}}$ increase (Fig. $5 C, D$ ). This is consistent with the classical idea that changes in mEPSC frequency represent a presynaptic locus, whereas changes to individual mEPSC size represent a postsynaptic locus. mEPSC $10-90 \%$ rise time $(1.08 \pm 0.07 \mathrm{~ms}$ for control and $1.24 \pm 0.07 \mathrm{~ms}$ for $\left.\mathrm{K}^{+} ; p<0.103\right)$ and $10-90 \%$ decay time $(2.21 \pm 0.08 \mathrm{~ms}$ for control and $2.15 \pm 0.08 \mathrm{~ms}$ for $\left.\mathrm{K}^{+} ; p<0.622\right)$ were also unchanged after chronic mild depolarization.

Because chronic mild depolarization appeared to alter glutamate signaling presynaptically, we wanted to verify that increased spiking alone could exact the same depression. To address this question, we electrically stimulated neurons at $10 \mathrm{~Hz}$ for $16 \mathrm{~h}$ using field stimulation. After this treatment, sucrose-evoked currents were recorded (Fig. $6 \mathrm{~A}$ ). As with chronic mild $\mathrm{K}^{+}$depolarization (Fig. 5), chronic spiking, albeit on a shorter time scale, depressed the size of the RRP (Fig. 6A,B). Sucrose-elicited mEPSCs also showed a reduced frequency (Fig. $6 B$ ), again consistent with the idea that a presynaptic change underlies this signaling deficit. After $10 \mathrm{~Hz}$ stimulation, mEPSC amplitude (19.4 $\pm 0.4 \mathrm{pA}$ for control and $19.0 \pm 0.7 \mathrm{pA}$ for stimulated), $10-90 \%$ rise time $(1.38 \pm 0.14 \mathrm{~ms}$ for control and $1.35 \pm 0.13 \mathrm{~ms}$ for stimulated; $p<0.900)$, and $10-90 \%$ decay time $(2.37 \pm 0.11$ $\mathrm{ms}$ for control and $2.57 \pm 0.10 \mathrm{~ms}$ for stimulated; $p<0.468) \mathrm{did}$ not change. By comparison, $16 \mathrm{~h}$ of mild depolarization $(6 \mathrm{~mm}$ added $\mathrm{K}^{+}$) produced no change in evoked EPSC amplitude ( $88 \pm$ $15 \%$ of control; $n=10 ; p<0.75)$.

Tests for changes in vesicle docking and priming

Both imaging and electrophysiological results were consistent with a presynaptic change strongly participating in synaptic adaptation to prolonged mild changes in physiological spiking. The results suggested elimination of vesicles in the RRP by functional
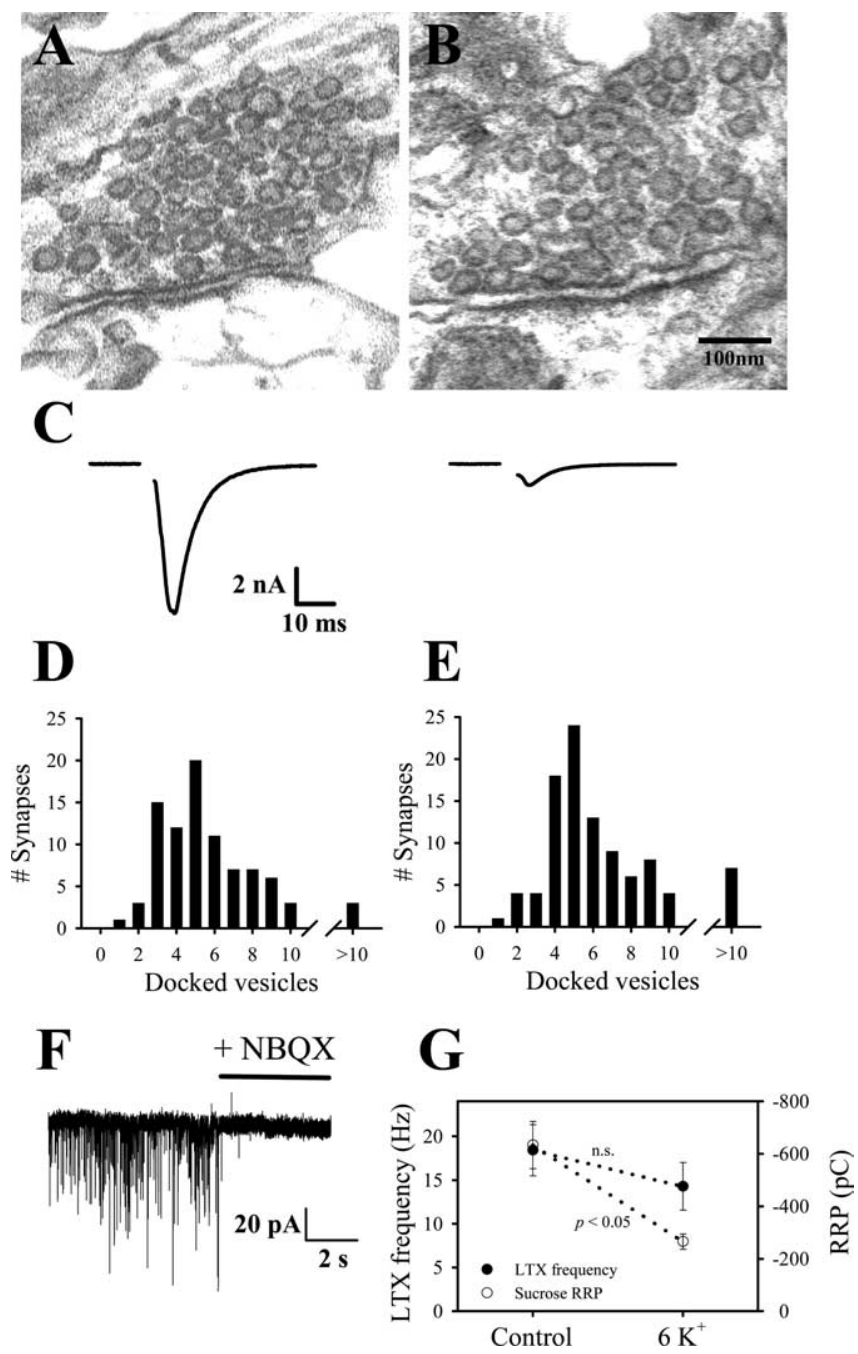

Figure 7. Chronic mild depolarization does not alter synapse ultrastructure, but rather alters synaptic vesicle priming. $A, B$, Electron micrographs of representative synapses from a control neuron $(\boldsymbol{A})$ or from a $\mathrm{K}^{+}$-treated neuron $(\boldsymbol{B})$. $\boldsymbol{C}$, EPSCs recorded from the control neuron whose synapse is shown in $\boldsymbol{A}$ (left) and from the $\mathrm{K}^{+}$-treated neuron whose synapse is shown in $\boldsymbol{B}$ (right). $\boldsymbol{D}-\boldsymbol{E}$, Histograms of the number of docked vesicles in synapses from control $(\boldsymbol{D})$ and $\mathrm{K}^{+}$-treated neurons $(\boldsymbol{E})$. For $\boldsymbol{D}$ and $\boldsymbol{E}, 88$ synapses (control) and 98 synapses $\left(\mathrm{K}^{+}\right)$were examined from three experiments. $\boldsymbol{F}$, Representative current trace of mEPSCs induced by $\alpha$-LTX application. The transient currents are mediated by AMPA receptors, because they are blocked with $1 \mu \mathrm{M} N B Q X$. G, Summary of the effect of chronic mild stimulation on the frequency of mEPSCs induced by $\alpha$-LTX application. No statistically significant difference was observed (filled circles). However, chronic mild stimulation depressed sucrose-evoked currents (open circles) recorded from the same neurons before $\alpha$-LTX application $(n=12)$. Error bars represent SEM.

inactivation of presynaptic terminals. The RRP has been associated with the complement of docked vesicles at presynaptic active zones (Schikorski and Stevens, 2001). Therefore, one hypothesis to explain the depression of release ready vesicles is that cells challenged by increased activity levels have fewer or no docked vesicles. We examined this ultrastructural question by exposing neurons to an additional $6 \mathrm{~mm}\left[\mathrm{~K}^{+}\right]_{\mathrm{o}}$ for $6-9 \mathrm{~d}$, then evoking autaptic postsynaptic responses (Fig. $7 C$ ), followed by electron microscopy of the same cells (Fig. $7 A, B$ ). We examined 88 random presynaptic terminals from control cultures and 98 terminals from depolarized cultures, but found no differences in the number of docked ( $5.57 \pm 0.59$ for control; $6.04 \pm 0.43$ for $\mathrm{K}^{+}$; $p<0.555)$ or total vesicles (55.73 \pm 0.63 for control; $67.59 \pm 7.87$ for $\left.\mathrm{K}^{+} ; p<0.208\right)$ in the terminals from these two groups. The 
percentage of the total vesicle number that was docked also remained unchanged (data not shown).

We considered several possible sources of type II error (failure to detect a difference between control and treated synapses). First, light microscopy (FM1-43/vGluT-1 comparisons) suggests that as few as $20 \%$ of terminals may experience an all-or-none loss of docked vesicles (Fig. 3C). A power analysis, performed to determine whether our sample sizes should have detected a loss of vesicles in $20 \%$ of synapses, demonstrated a power of $>99.9 \%$, indicating a type II error is highly unlikely. We also considered the possibility that although functional effects appeared all-ornone (Fig. 3D-F), morphological changes to the number of docked vesicles might be more subtle. As another test for a graded change in the number of vesicles docked per synapse, we performed a $\chi^{2}$ analysis to examine whether treated synapses had more synapses with fewer than three (less than one-half the average of control) docked vesicles. We found no difference between control and experimental groups in the number of synapses containing fewer than three vesicles $(p<0.125)$. Histograms of the numbers of docked vesicles per synapse illustrate that chronic mild depolarization did not increase the number of synapses with few docked vesicles (Fig. $7 D, E$ ).

Finally, the data shown in Figure 7 use a definition of docked vesicles as those vesicles that are apposed to the postsynaptic density and within one vesicle diameter from the plasma membrane (Pozzo-Miller et al., 1999; Murphy et al., 2000). If we used a stricter definition of docked, counting only vesicles in contact with the plasma membrane (Harris and Sultan, 1995; Collin et al., $2001)$, the number of docked vesicles decreased ( $4.84 \pm 0.52$ for control; $4.76 \pm 0.30$ for $\mathrm{K}^{+}$), but there was still no statistically significant difference between control and $\mathrm{K}^{+}$-treated neurons $(p<0.895)$. Therefore, a change in morphologically docked vesicles does not explain persistent RRP adaptation.

Genetic deletion of certain components of the synaptic machinery, such as munc13-1 at glutamate synapses, is associated with a decrease in the total cellular available vesicle pool and a decrease in functional synaptic terminals, but no ultrastructural change (Augustin et al., 1999; Rosenmund et al., 2002). A hallmark feature of the synaptic phenotype is that although neither action potentials nor hypertonic sucrose can evoke transmitter release, $\alpha$-LTX, the active component of black widow spider venom, is capable of evoking release of the dormant vesicles (Augustin et al., 1999). The interpretation is typically that vesicles in these mutants are stalled at a morphologically docked, but biochemically immature, state and thus are insufficiently primed for release (Augustin et al., 1999). Because activity-adapted vesicles appear still to be morphologically docked (Fig. 7), we might predict that these vesicles could be recruited to fuse by $\alpha$-LTX. Such a result would implicate a change in vesicle priming as the mechanism of adaptation.

To test whether physiological activity alters the percentage of functional presynaptic terminals by depressing vesicle priming, we challenged autaptic cells with sucrose to confirm the persistent RRP adaptation, then with $\alpha$-LTX to probe for docked but unprimed vesicles (Fig. $7 F$ ). Although sucrose responses showed the characteristic RRP depression, the frequency of mEPSCs evoked by $\alpha$-LTX was not statistically different in depolarized versus control neurons (Fig. $7 G)(p<0.451)$. These results further exclude a deficiency in postsynaptic function as a contributor to homeostatic depression and strongly implicate a deficiency in priming as the mechanism of expression of the presynaptic change.

\section{Discussion}

Homeostatic plasticity is an important means by which neurons can adjust their overall level of activity in the face of changing input strength. We showed previously that pathophysiological elevations in $\left[\mathrm{K}^{+}\right]_{\mathrm{o}}$ depolarize hippocampal neurons and subsequently depress glutamatergic signaling, presumably acting as a form of homeostatic regulation (Moulder et al., 2003, 2004). The locus of this depression was the presynaptic vesicular RRP (Moulder et al., 2004). In the present study, we found that physiological activity altered glutamate neurotransmission over a time scale of days. Again, the primary target of the activitydependent modulation was presynaptic, suggesting that RRP size (and/or availability) is a key factor in plastic changes to synaptic function. Furthermore, the response to sustained activity resulted in an apparent all-or-none regulation of presynaptic function, an unusual mechanism that violates the canonical idea that synaptic homeostasis regulates synaptic gain across all synaptic contacts (Turrigiano et al., 1998).

\section{Expression of synaptic adaptation}

Several pieces of evidence were consistent with our conclusion that chronic mild depolarization (like shorter, strong depolarizations) (Moulder et al., 2004) depressed glutamatergic signaling chiefly by affecting presynaptic function. We found no change in responses to exogenous AMPA/KA agonists, no change in mEPSC amplitude, and no change in the labeling intensity or size of postsynaptic receptor subunits. Instead, either silencing or increasing spiking from baseline modulated the percentage of FM1-43-positive synapses, providing strong evidence for a presynaptic adaptation. Specifically, the mismatch between functionally active presynaptic terminals and presence of vesicle markers points to a decrease in the number of active presynaptic sites and a change in the size of the total cellular RRP.

The fact that glutamate synapses were ultrastructurally normal in terms of total and docked vesicle number (Fig. 7A-E) seems to contradict the FM1-43 and electrophysiology results. However, the observation that $\alpha$-LTX, but not hypertonic sucrose, induced release of synaptic vesicles at these sites (Fig. 7G) reconciles the two experiments. Hippocampal neurons from mice genetically deleted for Munc13-1, a protein required for the transition between synaptic vesicle docking and priming, show the same selective response to $\alpha$-LTX (Augustin et al., 1999). We therefore conclude that priming is likely the target of synaptic adaptations to chronic changes in activity. Interestingly, neurons from mice genetically deleted for other presynaptic proteins, including the cytomatrix component bassoon, also display a similar phenotype (Altrock et al., 2003). Because priming is a critical step in the synaptic vesicle cycle, the idea that electrical activity could modulate priming has already been suggested (Dulubova et al., 2005), and a role for priming has been shown for short-term synaptic plasticity (Junge et al., 2004). To our knowledge, however, the current work represents the first report of priming being implicated in longer term, adaptive alterations in synaptic function.

After mild depolarization, the percentage of inactivated terminals detected by FM1-43/vGluT-1 comparisons seemed less prominent than changes to electrophysiologically defined RRP (compare Figs. 3C, 5D). Several factors may underlie this quantitative discrepancy. It is possible that graded changes in RRP size at individual terminals could contribute to electrophysiological results but fail to be detected by FM1-43 intensity analyses. This seems unlikely because there was no consistent change in the integrated intensities of active synapses after mild depolarization 
(Fig. $3 E, F$ ), and we were able to detect graded changes in intensity induced by TTX. It is also possible that synaptic adaptation involves downregulation of vGluT-1 (De Gois et al., 2005), which might depress EPSCs but not FM1-43 staining. This is unlikely because mEPSC amplitude was unchanged, and we detected no alteration in vGluT-1 staining intensity in depolarized cultures. There could be an undetected postsynaptic contribution to the electrophysiological studies, although several results (see below) make this unlikely. Finally, there could be effects of prolonged depolarization on total number of synaptic contacts formed, an effect that would be detected by several electrophysiological assays but not by our imaging assay. However, this hypothesis cannot explain the similarity of $\alpha$-LTX-evoked mEPSC frequencies in control and depolarized cultures (Fig. 7G). In summary, we suggest that small contributions of several of these mechanisms must add to explain the quantitative discrepancy.

\section{Requirements for induction}

Our initial experiments were performed using chronic exposure to an additional $6 \mathrm{mM}\left[\mathrm{K}^{+}\right]_{\mathrm{o}}$. This treatment brought neurons' membrane potential closer to threshold and increased spiking. Given that even subtle changes in presynaptic resting membrane potential can cause profound effects on $\left[\mathrm{Ca}^{2+}\right]_{\mathrm{i}}$ (Awatramani et al., 2005), we sought to determine the role of spiking in depression of glutamate neurotransmission. TTX alone increased the percentage of FM1-43-positive active terminals and prevented the decrease in the percentage of active terminals induced by prolonged mild depolarization. In addition, field stimulation to induce spiking directly also depressed the physiologically defined RRP. Together, these results demonstrate that physiological spiking activity is sufficient to induce presynaptic adaptation.

Interestingly, field stimulation at $10 \mathrm{~Hz}$ caused synaptic adaptation more rapidly (within $16 \mathrm{~h}$ ) than mild depolarization. These results are consistent with the general pattern in which strong, pathophysiological depolarization $\left(60 \mathrm{mM}\left[\mathrm{K}^{+}\right]_{\mathrm{o}}\right)$ induces presynaptic adaptation in $<1 \mathrm{~h}$ (Moulder et al., 2004) but lower levels of depolarization $\left(30 \mathrm{mM} \mathrm{K}^{+}\right)$require more time to induce detectable adaptation (Moulder et al., 2004). Therefore, persistent RRP depression can be induced by a wide range of stimuli at rates depending on stimulus strength.

\section{Comparison with previous results}

Our current results have focused on a presynaptic homeostatic change after both chronic activity blockade (Fig. 1) and a chronic upregulation of activity. As detailed above, several lines of experimentation were consistent with the idea that this presynaptic change is the primary locus for the homeostatic regulation in our system. However, controversy exists in the field as to whether chiefly presynaptic (Bacci et al., 2001; Shi et al., 2001; Moulder et al., 2004; De Gois et al., 2005; Wang et al., 2005) or postsynaptic (O’Brien et al., 1998; Turrigiano et al., 1998; Leslie et al., 2001; Ehlers, 2003; Ju et al., 2004; Perez-Otano and Ehlers, 2005; Richards et al., 2005; Wierenga et al., 2005) mechanisms, or even both (Luthi et al., 2001; Murthy et al., 2001; Alder et al., 2005; Thiagarajan et al., 2005), govern homeostasis. It is unclear why different studies on this question have led to different results. Potential reasons include the methodology (e.g., vital fluorescent dyes, electrophysiology), the neuronal system, the developmental stage (Burrone et al., 2002; Kirov et al., 2004), and the length of treatment. The last may be of particular interest because in the case of brain-derived neurotrophic factor-induced synaptic plasticity, early changes are reported to affect presynaptic machinery, whereas late changes affect postsynaptic components (Alder et al., 2005).

Another possible contributing factor is whether homeostasis in response to increased activity or to activity blockade is being examined. Furthermore, the means by which activity has been modulated can also differ (e.g., TTX treatment vs glutamate receptor blockade). Our current results do not exclude a possible postsynaptic contribution to homeostasis after activity blockade. Instead, they argue that presynaptic alterations, specifically to the functional RRP, do participate in the subsequent increase in synaptic function (Fig. 1). This may seem to be in contrast with recent work showing no alterations in FM1-43 uptake after activity blockade (Wierenga et al., 2005). However, the difference we demonstrate in Figure 1 is in the percentage of active (FM1-43positive) synapses, which may not have been detected in the previous work.

Although specific upregulation of synaptic GluR1 has been reported with chronic activity blockade (Ju et al., 2004; Thiagarajan et al., 2005), we did not observe any differences in GluR2 or GluR1 localization with chronic mild depolarization. Consistent with this, we did not find any difference in mEPSC kinetics, either with $\mathrm{K}^{+}$treatment or field stimulation (Figs. 5, 6), that would suggest an alteration in the synaptic receptor subunit composition. Therefore, it appears that modulation of GluR1 trafficking may be unique to homeostasis after activity blockade.

In sum, we found that even mild elevations in $\left[\mathrm{K}^{+}\right]_{\mathrm{o}}$ that result in increased spiking can cause presynaptic alterations with prolonged exposure. A similar presynaptic effect has been reported recently in vivo with chronic stimulation at the neuromuscular junction (Belair et al., 2005), indicating that these mechanisms are likely to be important in many forms of synaptic plasticity. It will be of great interest to determine under what conditions synaptic vesicle priming assumes the primary role in homeostatic modulation and to determine the molecular pathways involved in the induction and expression of this form of synaptic plasticity.

\section{References}

Ahmari SE, Buchanan J, Smith SJ (2000) Assembly of presynaptic active zones from cytoplasmic transport packets. Nat Neurosci 3:445-451.

Alder J, Thakker-Varia S, Crozier RA, Shaheen A, Plummer MR, Black IB (2005) Early presynaptic and late postsynaptic components contribute independently to brain-derived neurotrophic factor-induced synaptic plasticity. J Neurosci 25:3080-3085.

Altrock WD, tom Dieck S, Sokolov M, Meyer AC, Sigler A, Brakebusch C, Fassler R, Richter K, Boeckers TM, Potschka H, Brandt C, Loscher W, Grimberg D, Dresbach T, Hempelmann A, Hassan H, Balschun D, Frey JU, Brandstatter JH, Garner CC, et al. (2003) Functional inactivation of a fraction of excitatory synapses in mice deficient for the active zone protein bassoon. Neuron 37:787-800.

Augustin I, Rosenmund C, Sudhof TC, Brose N (1999) Munc13-1 is essential for fusion competence of glutamatergic synaptic vesicles. Nature 400:457-461.

Awatramani GB, Price GD, Trussell LO (2005) Modulation of transmitter release by presynaptic resting potential and background calcium levels. Neuron 48:109-121.

Bacci A, Coco S, Pravettoni E, Schenk U, Armano S, Frassoni C, Verderio C, De Camilli P, Matteoli M (2001) Chronic blockade of glutamate receptors enhances presynaptic release and downregulates the interaction between synaptophysin-synaptobrevin-vesicle-associated membrane protein 2. J Neurosci 21:6588-6596.

Belair EL, Vallee J, Robitaille R (2005) Long-term in vivo modulation of synaptic efficacy at the neuromuscular junction of Rana pipiens frogs. J Physiol (Lond) 569:163-178.

Burrone J, Murthy VN (2003) Synaptic gain control and homeostasis. Curr Opin Neurobiol 13:560-567.

Burrone J, O’Byrne M, Murthy VN (2002) Multiple forms of synaptic plas- 
ticity triggered by selective suppression of activity in individual neurons. Nature 420:414-418.

Collin C, Vicario-Abejon C, Rubio ME, Wenthold RJ, McKay RD, Segal M (2001) Neurotrophins act at presynaptic terminals to activate synapses among cultured hippocampal neurons. Eur J Neurosci 13:1273-1282.

De Gois S, Schafer MK, Defamie N, Chen C, Ricci A, Weihe E, Varoqui H, Erickson JD (2005) Homeostatic scaling of vesicular glutamate and GABA transporter expression in rat neocortical circuits. J Neurosci 25:7121-7133.

Dulubova I, Lou X, Lu J, Huryeva I, Alam A, Schneggenburger R, Sudhof TC, Rizo J (2005) A Munc13/RIM/Rab3 tripartite complex: from priming to plasticity? EMBO J 24:2839-2850.

Ehlers MD (2003) Activity level controls postsynaptic composition and signaling via the ubiquitin-proteasome system. Nat Neurosci 6:231-242.

Gao TM, Howard EM, Xu ZC (1998) Transient neurophysiological changes in CA3 neurons and dentate granule cells after severe forebrain ischemia in vivo. J Neurophysiol 80:2860-2869.

Gao TM, Pulsinelli WA, Xu ZC (1999) Changes in membrane properties of CA1 pyramidal neurons after transient forebrain ischemia in vivo. Neuroscience 90:771-780.

Harata N, Pyle JL, Aravanis AM, Mozhayeva M, Kavalali ET, Tsien RW (2001) Limited numbers of recycling vesicles in small CNS nerve terminals: implications for neural signaling and vesicular recycling. Trends Neurosci 24:637-643.

Harris KM, Sultan P (1995) Variation in the number, location and size of synaptic vesicles provides an anatomical basis for the nonuniform probability of release at hippocampal CA1 synapses. Neuropharmacology 34:1387-1395.

Ju W, Morishita W, Tsui J, Gaietta G, Deerinck TJ, Adams SR, Garner CC, Tsien RY, Ellisman MH, Malenka RC (2004) Activity-dependent regulation of dendritic synthesis and trafficking of AMPA receptors. Nat Neurosci 7:244-253.

Junge HJ, Rhee JS, Jahn O, Varoqueaux F, Spiess J, Waxham MN, Rosenmund C, Brose N (2004) Calmodulin and Munc13 form a $\mathrm{Ca}^{2+}$ sensor/effector complex that controls short-term synaptic plasticity. Cell 118:389-401.

Kay AR, Alfonso A, Alford S, Cline HT, Holgado AM, Sakmann B, Snitsarev VA, Stricker TP, Takahashi M, Wu LG (1999) Imaging synaptic activity in intact brain and slices with FM1-43 in C. elegans, lamprey, and rat. Neuron 24:809-817.

Kirov SA, Goddard CA, Harris KM (2004) Age-dependence in the homeostatic upregulation of hippocampal dendritic spine number during blocked synaptic transmission. Neuropharmacology 47:640-648.

Leslie KR, Nelson SB, Turrigiano GG (2001) Postsynaptic depolarization scales quantal amplitude in cortical pyramidal neurons. J Neurosci 21:RC170(1-6).

Luthi A, Schwyzer L, Mateos JM, Gahwiler BH, McKinney RA (2001) NMDA receptor activation limits the number of synaptic connections during hippocampal development. Nat Neurosci 4:1102-1107.

Mennerick S, Que J, Benz A, Zorumski CF (1995) Passive and synaptic properties of hippocampal neurons grown in microcultures and in mass cultures. J Neurophysiol 73:320-332.

Moulder KL, Fu T, Melbostad H, Cormier RJ, Isenberg KE, Zorumski CF, Mennerick S (2002) Ethanol-induced death of postnatal hippocampal neurons. Neurobiol Dis 10:396-409.
Moulder KL, Cormier RJ, Shute AA, Zorumski CF, Mennerick S (2003) Homeostatic effects of depolarization on $\mathrm{Ca}^{2+}$ influx, synaptic signaling, and survival. J Neurosci 23:1825-1831.

Moulder KL, Meeks JP, Shute AA, Hamilton CK, de Erausquin G, Mennerick S (2004) Plastic elimination of functional glutamate release sites by depolarization. Neuron 42:423-435.

Murphy DD, Rueter SM, Trojanowski JQ, Lee VM (2000) Synucleins are developmentally expressed, and $\alpha$-synuclein regulates the size of the presynaptic vesicular pool in primary hippocampal neurons. J Neurosci 20:3214-3220.

Murthy VN, Schikorski T, Stevens CF, Zhu Y (2001) Inactivity produces increases in neurotransmitter release and synapse size. Neuron 32:673-682.

O’Brien RJ, Kamboj S, Ehlers MD, Rosen KR, Fischbach GD, Huganir RL (1998) Activity-dependent modulation of synaptic AMPA receptor accumulation. Neuron 21:1067-1078.

Perez-Otano I, Ehlers MD (2005) Homeostatic plasticity and NMDA receptor trafficking. Trends Neurosci 28:229-238.

Pozzo-Miller LD, Gottschalk W, Zhang L, McDermott K, Du J, Gopalakrishnan R, Oho C, Sheng ZH, Lu B (1999) Impairments in high-frequency transmission, synaptic vesicle docking, and synaptic protein distribution in the hippocampus of BDNF knockout mice. J Neurosci 19:4972-4983.

Richards DA, Mateos JM, Hugel S, de Paola V, Caroni P, Gahwiler BH, McKinney RA (2005) Glutamate induces the rapid formation of spine head protrusions in hippocampal slice cultures. Proc Natl Acad Sci USA 102:6166-6171.

Rosenmund C, Stevens CF (1996) Definition of the readily releasable pool of vesicles at hippocampal synapses. Neuron 16:1197-1207.

Rosenmund C, Sigler A, Augustin I, Reim K, Brose N, Rhee JS (2002) Differential control of vesicle priming and short-term plasticity by Munc13 isoforms. Neuron 33:411-424.

Schikorski T, Stevens CF (2001) Morphological correlates of functionally defined synaptic vesicle populations. Nat Neurosci 4:391-395.

Shi J, Aamodt SM, Townsend M, Constantine-Paton M (2001) Developmental depression of glutamate neurotransmission by chronic low-level activation of NMDA receptors. J Neurosci 21:6233-6244.

Sudhof TC (2004) The synaptic vesicle cycle. Annu Rev Neurosci 27:509-547.

Thiagarajan TC, Lindskog M, Tsien RW (2005) Adaptation to synaptic inactivity in hippocampal neurons. Neuron 47:725-737.

Turrigiano GG, Nelson SB (2004) Homeostatic plasticity in the developing nervous system. Nat Rev Neurosci 5:97-107.

Turrigiano GG, Leslie KR, Desai NS, Rutherford LC, Nelson SB (1998) Activity-dependent scaling of quantal amplitude in neocortical neurons. Nature 391:892-896.

Wang X, Li Y, Engisch KL, Nakanishi ST, Dodson SE, Miller GW, Cope TC, Pinter MJ, Rich MM (2005) Activity-dependent presynaptic regulation of quantal size at the mammalian neuromuscular junction in vivo. J Neurosci 25:343-351.

Wierenga CJ, Ibata K, Turrigiano GG (2005) Postsynaptic expression of homeostatic plasticity at neocortical synapses. J Neurosci 25:2895-2905.

Xu W, Cormier R, Fu T, Covey DF, Isenberg KE, Zorumski CF, Mennerick S (2000) Slow death of postnatal hippocampal neurons by $\mathrm{GABA}_{\mathrm{A}}$ receptor overactivation. J Neurosci 20:3147-3156. 